\title{
Bragg curve spectroscopy for improved fission fragment identification
}

\author{
Bryan Fulsom ${ }^{1, *}$ \\ ${ }^{1}$ Pacific Northwest National Laboratory, Richland WA 99352, USA
}

\begin{abstract}
We report on the development of Bragg curve spectroscopy techniques to improve fission fragment identification in the measurement of independent fission product yields. The NIFFTE collaboration's fissionTPC detector provides ionization energy and particle tracking information from neutroninduced fission targets. A joint effort between PNNL, LLNL, LANL, and the Colorado School of Mines is investigating the ionization profiles deposited by $\mathrm{U}-235$, U-238, and Pu-239 fission products in this detector, with the goal of including additional stopping power information beyond a standard $2 \mathrm{E}$ analysis. The aim is to improve the determination of fragment atomic and mass numbers with this information, via methods such as parametric fits and machine learning techniques.
\end{abstract}

\section{Introduction}

This talk focuses on fission product yield measurements made with the NIFFTE TPC detector. It first covers a description of the detector, then provides detailed information on the application of the $2 \mathrm{E}$ method to measure fission product yields from a ${ }^{235} \mathrm{U}$ target, with preliminary results. We then discuss the use of $d E / d x$ information afforded by the tracking capabilities of the NIFFTE TPC to improve fission product identification. Finally, the initial application of machine learning techniques to improve this effort is presented.

\section{The NIFFTE TPC}

The Neutron Induced Fission Fragment Tracking Experiment (NIFFTE) is a collaboration between seven U.S. institutions to construct the "fissionTPC" time projection chamber located at the Los Alamos Neutron Science Center (LANSCE). The detector was designed to perform precision measurements of fission cross section ratios using tracking capability to reduce systematic uncertainties. For a sample of recent NIFFTE collaboration publications see $[1-3]$.

The fissionTPC is a dual-volume gas time projection chamber (see Fig. 1). The anode detection planes consist of 2976 hex pad MicroMEGAS detectors with $2 \mathrm{~mm}$ pitch. The ionization gas volume is filled with a 95\% argon / 5\% isobutane mix, operated at 560 torr. The drift distance in the direction parallel to the neutron beam is $54 \mathrm{~mm}$. Signals are readout with a data acquisition system with $50 \mathrm{MHz}$ pad readout, and $1 \mathrm{GHz}$ for the cathode used

*e-mail: bryan.fulsom@pnnl.gov 


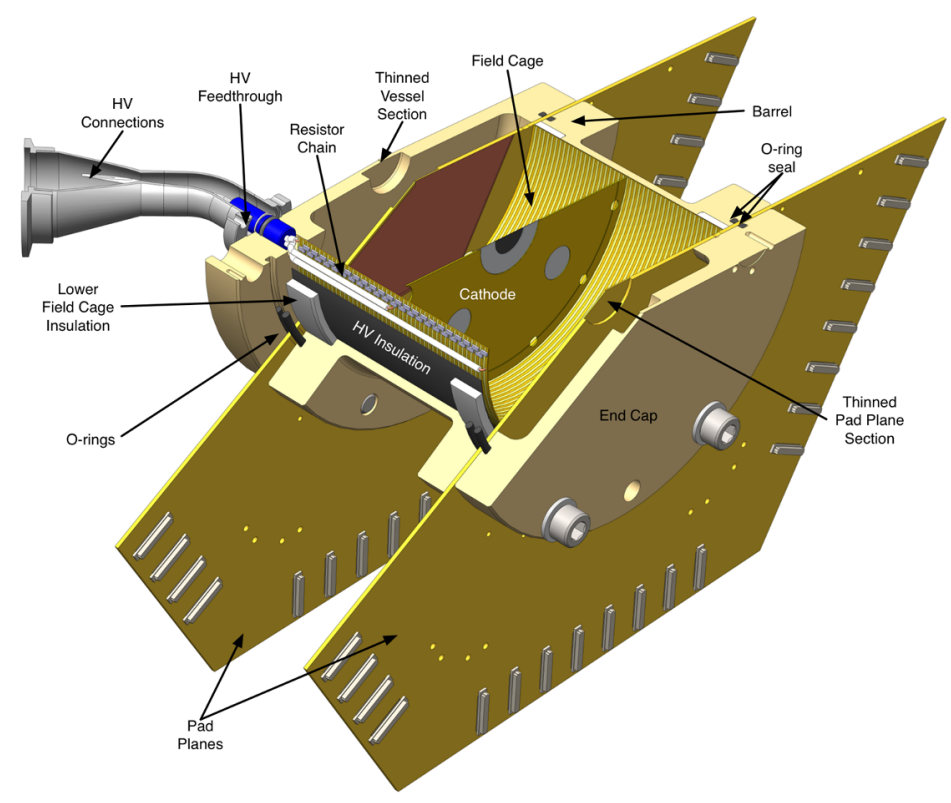

Figure 1. Schematic layout of the NIFFTE fissionTPC time projection chamber.

for neutron time-of-flight (energy) measurements. Publications are available detailing the NIFFTE detector [4], data acquisition [5], and operation [6].

The NIFFTE TPC is situated on the LANSCE Weapons Neutron Research facility 90L beamline, which provides an unmoderated source of neutrons ranging in energy from $\sim 0.1$ to $100 \mathrm{MeV}$ (though in practice, given the flux and region of interest, energies up to $O(10)$ $\mathrm{MeV}$ are considered). Here we consider data collected from the neutron beam on targets of ${ }^{235} \mathrm{U},{ }^{238} \mathrm{U}$, and ${ }^{239} \mathrm{Pu}$, taken during 2016 and 2017 . The $100 \mu \mathrm{g} / \mathrm{cm}^{2}$ targets were prepared at Oregon State University by vapor deposition on a thin $\left(\sim 100 \mu \mathrm{g} / \mathrm{cm}^{2}\right)$ carbon backing.

Time projection chambers provide the ability to fully reconstruct the three-dimensional path of particle tracks. From these tracks, one can measure the direction, energy, starting vertex position, and length. The energy loss per unit distance along the track (Bragg curve), $d E / d x$, can also be measured. The combination of these properties allows the NIFFTE TPC measurements to precisely select fission fragments eminating from mixed isotope targets, discriminate between proton, $\alpha$ particles, and fission fragments based on track length and energy, and potentially mass $(A)$ and nuclear charge $(Z)$ from details of the Bragg curve.

\section{Application of the 2E Method to NIFFTE TPC data}

This section provides details of the application of the "2E Method" to NIFFTE TPC data.

Events are considered for further study if there is at least one track reconstructed in both volumes, with a minimum ADC count (energy) of 3500, originating from the ${ }^{235} \mathrm{U}$ target location. The tracks must fall within $0.30<\cos \theta<0.92\left(23^{\circ}<\theta<73^{\circ}\right)$, where $\theta$ is the angle between the track path and the neutron beam axis. This selection removes fragments that pass through large amounts of target/backing material (steep $\theta$ ) and those whose signals 
become saturated in the DAQ system (shallow $\theta$ ). Finally, based on studies of anode pad efficiency, tracks entering regions of "dead" and low-efficiency pads are removed, in order to maintain the highest quality data sample.

Energy loss in the target and backing is the largest source of correction and systematic uncertainty in this analysis. A correction is applied according to the angle of the track, as a proxy for the amount of thickness of material traversed. The energy loss correction is estimated from a linear fit to the number of ADC counts versus $1 / \cos \theta$ for all tracks in the data set. Separate corrections are applied for each volume, and can range between 10-30 $\mathrm{MeV}$ for fission fragments passing through the target carbon backing.

The raw ADC count is converted to units of energy in $\mathrm{MeV}$ by scaling the total kinetic energy of the events to match the average of four literature values for ${ }^{235} U$ [7-10]. A small linear volume-to-volume correction is applied based on the positions of the light and heavy fragment peaks. As a crosscheck, the total kinetic energy found in the NIFFTE TPC data versus neutron energy is compared against three literature values $[10,12,13]$. The mean value most closely matches those from GEF simulation [12], but the energy resolution is found to be greater than $13 \mathrm{MeV}$, which is several $\mathrm{MeV}$ worse than other measurements.

A full description of the "2E Method" for determining fission product yields can be found in a separate talk at this workshop [11]. Aside from the energy corrections, the mechanics of the iterative method are unchanged by the detector type and can be applied equally to the NIFFTE TPC. Preliminary results for ${ }^{235} \mathrm{U}$ fission product yield versus neutron energy are presented in Fig. 2. The yields are comparable to the known England and Rider standard [14], but the mass resolution of these results were found to be greater than $\sim 8$ a.m.u., which is substantially broader.

The next stage of development for the 2E method with NIFFTE is to focus on improving the largest areas of systematic uncertainty. An improved model-based energy loss correction in material will be implemented. Effects of "pulse height defect", i.e., ionization energy losses of any type in the detector, will be studied, and could be corrected using an approximation based on empirical values [15] or from ion beam measurements in fissionTPC drift gas. The uncertainty due to estimation of neutron evaporation in the fission process (common to the $2 \mathrm{E}$ method in all detectors) can be assessed by considering alternate $v(A)$ models. Finally, the tracking ability of the TPC can be specifically exploited by using accurately-measured track length in place of energy (which has worse resolution), and by considering ionization profile information.

\section{Use of $d E / d x$ profiles for improved fission fragment identification}

As mentioned in Section 2, the TPC potentially gives us further track-related information, such as energy, length, and Bragg peak height and position. However, it is important to note that the general shape of the Bragg curve for fission products is quite different than that one might expect from $\alpha$ particles. We use the SRIM and TRIM software packages [16] to simulate and parameterize the energy loss curves expected for fission products passing through our gas volume. From other studies [17], we expect this to provide a reasonable agreement between the simulation and reality.

We consider two starting points based on SRIM when practically modeling the expected Bragg curves in our data: interpolation of SRIM look-up tables spanning a large range of $A, Z$, and $E$, and a parametrization using an empirical function fitted to SRIM outputs. In both cases, we estimate the amount of nuclear energy losses appearing as ionization based on a fit to TRIM simulations. Then we apply a data-determined offset and Gaussian smearing to these idealized curves to model how these curves would appear in our data. In order to 


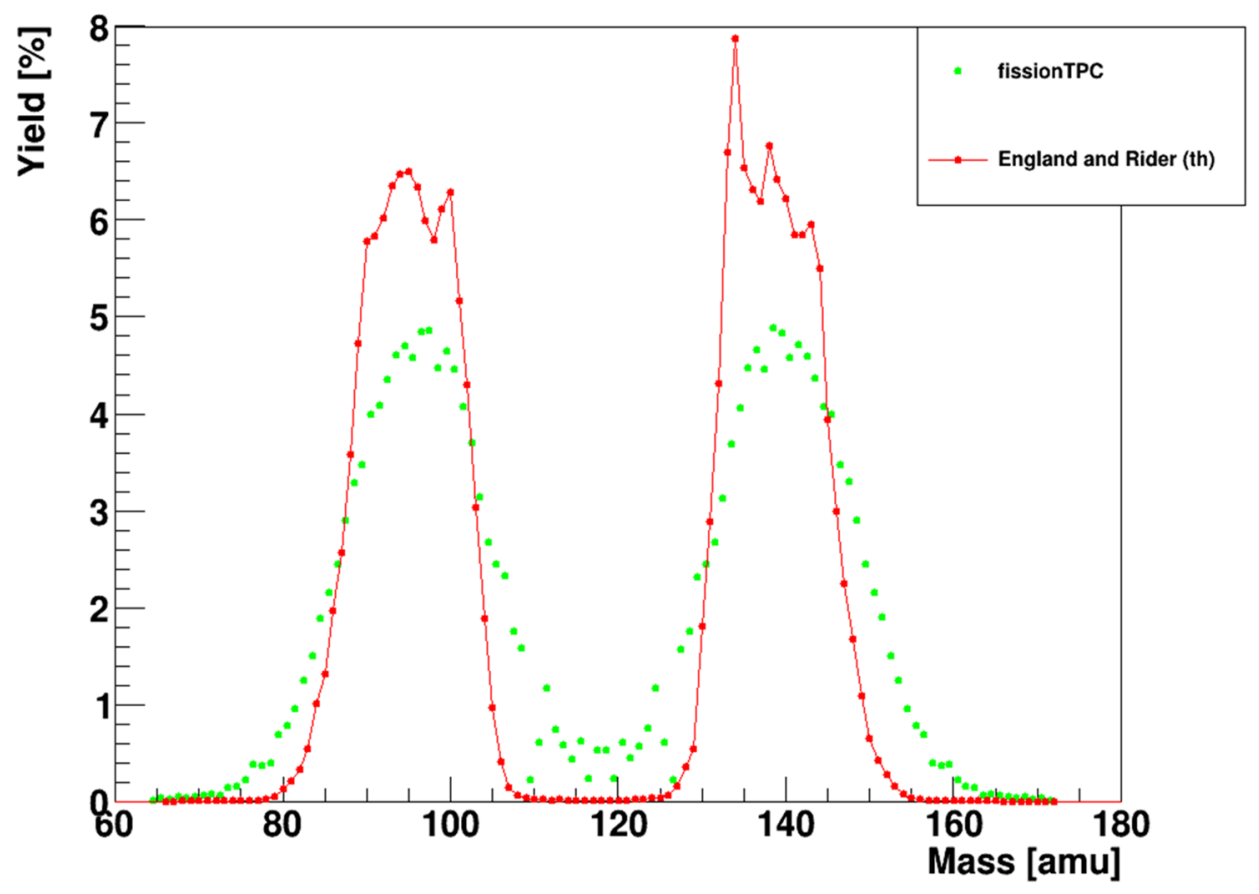

Figure 2. Preliminary ${ }^{235} \mathrm{U}$ fission product yield measurements using the $2 \mathrm{E}$ method with NIFFTE TPC data for incident neutron energies of $0.18<E_{n}<0.20 \mathrm{MeV}$. The green points are values derived from data, the red connected line represents the literature reference [14].

determine the value of $A$ and $Z$ for a given event, we minimize the $\chi^{2}$ difference between the Bragg curve in the data and that from our model.

A representative Bragg curve fit result is shown in Fig. 3. Preliminary results on ${ }^{235} \mathrm{U}$ data using solely $d E / d x$ Bragg curve information give qualitative indications for light and heavy fission fragments clustered in $A$ and $Z$, but also clear indications of pathological and non-physical results (Fig. 4) that remain to be understood.

We intend several avenues of investigation in order to improve these results. Since the 2E Method can effectively provide a value for $A$ (to which Bragg curve measurements are relatively insensitive), it can be used as constraint on the Bragg curve fit to provide a value for $Z$. The basis of the $d E / d x$ curves could also benefit from improvements, e.g., better understanding of tracking/ionization pulse height defect, alternative models to SRIM, or potentially restriction of the analysis to well-known regions of the curve. A more complicated but potentially useful approach could be to implement machine learning techniques for event and curve classification.

Machine learning techniques have been typically applied with great success in image identification applications. In this case we would use points along the Bragg curve distribution as inputs, with neural network training to output values of $A$ and $Z$. If we cannot determine values of $A$ and $Z$ outright with machine learning, we might at least discover the most relevant regions or metrics for Bragg curve identification. Another possible application could be identification and removal of events with poor tracking, low detector efficiency, etc. 


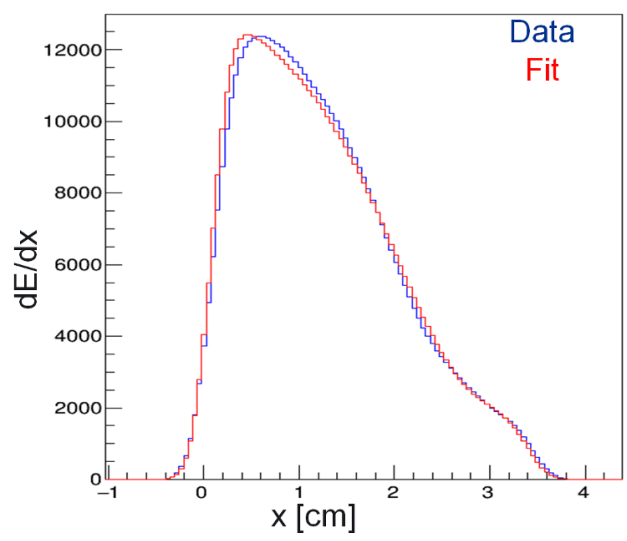

Figure 3. An illustrative example of a measured Bragg curve and the curve resulting from the fit minimization.
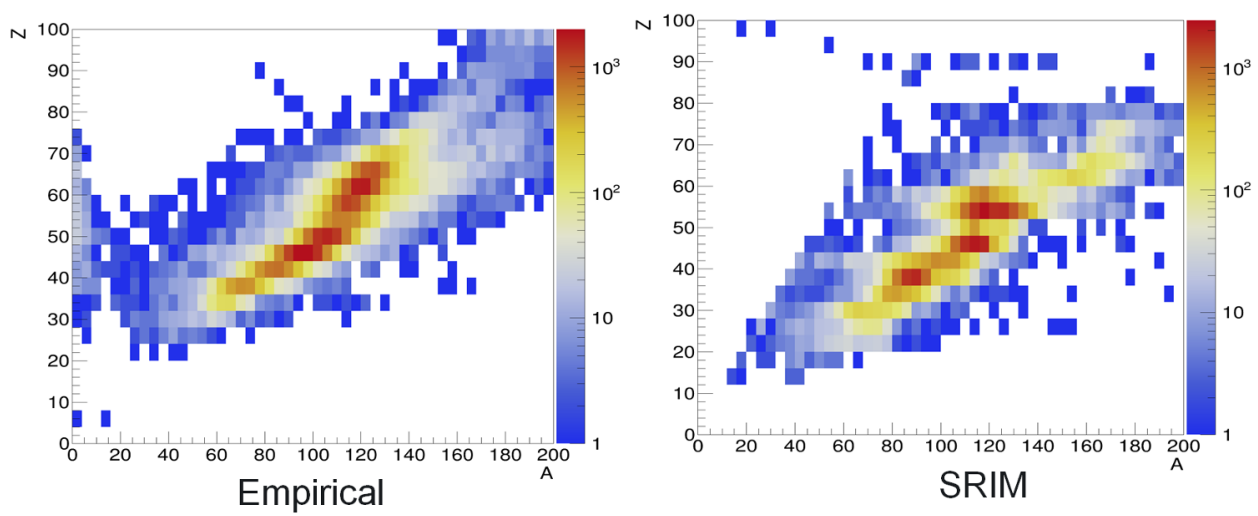

Figure 4. Preliminary ${ }^{235} U$ yields of $Z$ versus $A$ from Bragg curve fits. The left plot uses an empirical estimate based on SRIM, while the right plot uses interpolated SRIM look-up tables. In both cases qualitative indications for heavy and light fission fragment regions are present, but are overshadowed by pathological and unphysical results.

In any case, one of the primary concerns with using machine learning is the ability to provide explainations for the decisions made, and to attribute uncertainties in a proper manner.

As a preliminary investigation, we have produced a neural network based upon Keras [18] and TensorFlow [19], which takes a toy model for Bragg curves using simple mathematical functions and outputs a decision based on training of four different sample curves. The model takes 20 points along the sample Bragg curve as input, with varied offset and scaling for each curve. Within this test exercise, we find a $99.7 \%$ classification accuracy. The next step is to generate a more realistic training data set using TRIM output convolved with detector resolution effects. This training can also include other variables, such as track length, and Bragg peak energy and location, in the list of inputs. An underlying principle is to understand the relative importance of each input node towards the final classification decision, in order to gain a greater understanding of the underlying physics information. This might be achieved 
through the use of software tools such as LIME (Local Interpretable Model-Agnostic Explanations) [20].

\section{Conclusions}

This talk has presented the starting phases of an analysis of ${ }^{235} \mathrm{U}$ fission product yields based on data collected by the NIFFTE collaboration fissionTPC. The NIFFTE detector provides complete $3 \mathrm{D}$ tracking information, including $d E / d x$, which is potentially useful for identification of $A$ and $Z$ mass numbers. Based on the iterative $2 \mathrm{E}$ method, working initial results consistent with previous measurements have been obtained, and focus has now shifted to understanding and reducing the associated uncertainties. We have begun to integrate ionization profile information into the data analysis, and are investigating the advantages of using machine learning techniques to further improve discriminatory power available by exploiting the full fission event information.

\section{References}

[1] R. J. Casperson et al. (NIFFTE Collaboration), Phys. Rev. C 97, 034618 (2018).

[2] V. Geppert-Kleinrath et al. (NIFFTE Collaboration), Phys. Rev. C 99, 064619 (2019).

[3] N. I. Walsh et al. (NIFFTE Collaboration), arXiv:1904.10558 (2019).

[4] M. Heffner et al. (NIFFTE Collaboration), Nucl. Instr. and Meth. A 759, 50 (2014).

[5] M. Heffner, V. Riot, and L. Fabris, IEEE Trans. Nucl. Sci. NS-60, 2196 (2014).

[6] L. Snyder et al. (NIFFTE Collaboration), Nucl. Instr. and Meth. A 881, 1 (2018).

[7] J. C. D. Milton and J. S. Fraser, Can. J. Phys. 40, 1626 (1962).

[8] H. W. Schmitt, J. N. Neiler, and F.J. Walter, Phys. Rev. 141, 1146 (1966).

[9] M. J. Bennett and W. E. Stein, Phys. Rev. 156, 1277 (1967).

[10] D. L. Duke, Ph.D. thesis, LA-UR-15-28829 (2015).

[11] For a more complete discussion of the 2E Method, e.g. please see the talk by D. L. Duke at this workshop.

[12] K.-H. Schmidt et al., Nucl. Data Sheets 131107 (2016).

[13] R. Yanez et al., Nucl. Phys. A 970, 65 (2018).

[14] T. R. England and B. F. Rider, LA-UR-94-3106 (1994).

[15] F.-J. Hambsch, J. van Aarle, and R. Vogt, Nucl. Instr. and Meth. A 361, 257 (1995).

[16] J. F. Ziegler, M. D. Ziegler, and J. P. Biersack, Nucl. Instr. and Meth. B 268, 1818 (2010).

[17] J. G. Hajnrych, P. Filliatre, and B. Geslot, Nucl. Instr. and Meth. A 911, 15 (2018).

[18] F. Chollet et al., https://keras.io (2015).

[19] M. Abadi et al., http://tensorflow.org/ (2015).

[20] M. T. Ribeiro, S. Singh, and C. Guestrin, arXiv:1602.04938 (2016). 